Pacific Journal of Mathematics

$D$-HARMONIC DISTRIBUTIONS AND GLOBAL
HYPOELLIPTICITY ON NILMANIFOLDS 


\title{
D-HARMONIC DISTRIBUTIONS AND GLOBAL HYPOELLIPTICITY ON NILMANIFOLDS
}

\author{
JACEK M. CYgAN AND LeONARD F. RichaRdSON
}

Let $M=\Gamma \backslash N$ be a compact nilmanifold. A system of differential operators $D_{1}, \ldots, D_{k}$ on $M$ is globally hypoelliptic (GH) if when $D_{1} f=g_{1}, \ldots, D_{k} f=g_{k}$ with $f \in \mathscr{D}^{\prime}(M), g_{1}, \ldots, g_{k} \in C^{\infty}(M)$ then $f \in C^{\infty}(M)$. Let $X_{1}, \ldots, X_{k}$ be real vector fields on $M$ induced by the Lie algebra $\mathscr{N}$ of $N$. We study the relationships between (GH) of the system $X_{1}, \ldots, X_{k}$ on $M,(\mathbf{G H})$ of the operator $D=X_{1}^{2}+\cdots+X_{k}^{2}$, the constancy of $D$-harmonic distributions on $M$, and related algebraic conditions on $X_{1}, \ldots, X_{k} \in \mathscr{N}$.

0. Introduction. Let $M=\Gamma \backslash N$ be a compact nilmanifold, where $N$ is a connected, simply connected real nilpotent Lie group with a discrete subgroup $\Gamma$. There is a unique probability measure $\mu$ defined on the Borel sets on $M$ and invariant under the action of $N$ on $M$ by right translations. Every $\mu$-integrable function $f$ on $M$ defines a distribution by the formula $(f, \phi)=\int_{M} f \phi d \mu, \phi \in C^{\infty}(M)$. Let $\mathscr{N}$ be the Lie algebra of $N$. If $X \in \mathscr{N}$ then $X$ induces a vector field (which we will denote also by $X$ ) on $\Gamma \backslash N$ by $(X f)(\Gamma n)=$ $\left.(d / d t)\right|_{t=0} f(\Gamma n \exp t X)$. Consider the left-invariant sum of squares of such vector fields $X_{1}, \ldots, X_{k} \in \mathscr{N}$. This second order differential operator $D=X_{1}^{2}+\cdots+X_{k}^{2}$ can be regarded as acting on the right on distributions on $\Gamma \backslash N$. A distribution $u \in \mathscr{D}^{\prime}(M)$ is $D$-harmonic if $D u=0$ on $M$. The operator $D$ is globally hypoelliptic $(\mathrm{GH})$ if when $D f=g$ with $f \in \mathscr{D}^{\prime}(M), g \in C^{\infty}(M)$, then $f \in C^{\infty}(M)$. The system of vector fields $X_{1}, \ldots, X_{k}$ on $M$ is $(\mathrm{GH})$ if when $X_{1} f=$ $g_{1}, \ldots, X_{k} f=g_{k}$ with $f \in \mathscr{D}^{\prime}(M), g_{1}, \ldots, g_{k} \in C^{\infty}(M)$, then $f \in C^{\infty}(M)$. In this paper we investigate relationships between $(\mathrm{GH})$ of $D,(\mathrm{GH})$ of the corresponding system $X_{1}, \ldots, X_{k}$ of vector fields, the constancy of $D$-harmonic distributions on $M$, and related algebraic conditions on $X_{1}, \ldots, X_{k} \in \mathscr{N}$.

Our results are summarized in the figure below. In this figure, functionals $\Lambda \in \mathscr{N}_{j}^{*}$ are assumed to be integral, i.e. $\Lambda\left(\log \Gamma \cap \mathscr{N}_{j}\right) \subseteq \mathbb{Z}$; $\mathscr{N}=\mathscr{N}_{1} \supset \mathscr{N}_{2} \supset \cdots \supset \mathscr{N}_{r} \supset \mathscr{N}_{r+1}=\{0\}$ is the lower central series of $\mathscr{N}$ (we say $\mathscr{N}$ is of step r), and $\mathscr{L}$ is the subalgebra of $\mathscr{N}$ Liegenerated by $X_{1}, \ldots, X_{k}$. Let $\mathscr{W}_{\pi}$ be an ideal in $\operatorname{ker}(d \pi)$ such that 
$\mathscr{N} / \mathscr{W}_{\pi}$ has one dimensional center on which $\pi$ is non-trivial. Then $\overline{\mathscr{L}}:=\mathscr{L}+\mathscr{W}_{\pi}$ and $\overline{\mathscr{Z}}:=\mathscr{Z}+\mathscr{W}_{\pi}$.

$$
\begin{gathered}
\Lambda\left(\left(\mathscr{L} \cap \mathscr{N}_{j}\right)+\mathscr{N}_{j+1}\right) \neq 0 \\
\forall \Lambda \in\left(\mathscr{N}_{j} / \mathscr{N}_{j+1}\right)^{*} \\
j=1, \ldots, r(2.2)
\end{gathered}
$$

$\Downarrow 2$

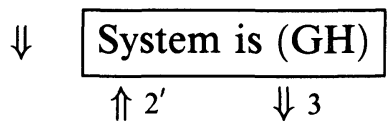

$\overline{\mathscr{L}} \cap \overline{\mathscr{Z}} \neq\{\overline{0}\}, \forall \pi \in(\Gamma \backslash N)_{\infty}$ (4.1)

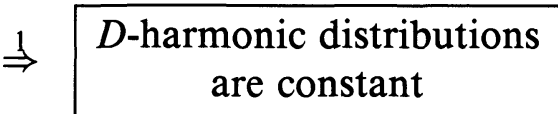

are constant

$$
\frac{\Downarrow}{\operatorname{dim} \operatorname{ker}(D)<\infty}
$$

$\stackrel{4}{\stackrel{4}{\leftrightarrows}}$

We explain below the labeled implications in the above figure referring the reader to indicated sections of the paper for details.

1. This is Theorem (2.1). Condition (2.2) with $j=1$ provides constancy of the $D$-harmonic distributions on the associated torus.

2. This holds with the necessary assumption that the system $X_{1}, \ldots, X_{k}$ is $(\mathrm{GH})$ on the associated torus (proved in [C-R2], Theorem 1).

$2^{\prime}$. This requires the assumption that the system $X_{1}, \ldots, X_{k}$ is $(\mathrm{GH})$ on the associated torus (implicitly contained in [C-R2] and discussed here in $\S 4$ ).

3. This is proved in $\S 4$ for $N$ with exclusively flat coadjoint orbits (which includes step 2 groups), and also for any nilpotent semidirect product $\mathbb{R} \ltimes \mathbb{R}^{n}$.

4. This is always true. (If $X_{1} f, \ldots, X_{k} f$ are $C^{\infty}$, then so is $D f=\left(X_{1}^{2}+\cdots+X_{k}^{2}\right) f$ and by $(\mathrm{GH})$ of $\left.D, f \in C^{\infty}.\right)$

5. We prove this converse to implication 4 for $N$ of step 2, if $D=X_{1}^{2}+X_{2}^{2}$ with $X_{1} \in \mathcal{N}, X_{2} \in \mathscr{N}_{2}$ and with a necessary growth condition on $X_{2}$ in $\S 1$. A growth condition on $X_{1}$ follows from $(\mathrm{GH})$ of the system $X_{1}, X_{2}$. Implication 5 is false for solvmanifolds, even if all the vector fields $X_{1}, \ldots, X_{k}$ are algebraic, and hence satisfy all growth conditions. Indeed, the example in $\S 3$ shows such a $D$ with a non- $L^{2}$ distribution in its kernel.

6. See e.g. [G-W3], Lemma 3, p. 161. 
We would like to thank L. Baggett, A. Hulanicki, J. Jenkins, and P. Ohring for many helpful discussions during the preparation of this paper.

1. 2-step nilmanifolds. In this section we show that global hypoellipticity of the system $X_{1}, \ldots, X_{k}$ is insufficient for $(\mathrm{GH})$ of $D$, even if $N$ is step 2, (Example (1.4)). Growth conditions on all the vector fields are needed. Under such conditions $D$ can be proven to be $(\mathrm{GH})$, at least on step 2 nilmanifolds (Theorem (1.1)). We'll see in $\S 3$ that this cannot happen in general solvmanifolds.

(1.1) THEOREM. Let $\mathscr{N}$ be a step 2 rational nilpotent Lie algebra, $N$ the corresponding connected, simply connected group, and $\Gamma$ a cocompact discrete subgroup of $N$. Let $Y_{1}, \ldots, Y_{n} ; Z_{1}, \ldots, Z_{k}$ be a linear basis for $\mathscr{N}$ selected from $\log \Gamma$ and such that $Y_{l}+[\mathscr{N}, \mathscr{N}]$, $l=1, \ldots, n$ is a basis of $\mathscr{N} /[\mathscr{N}, \mathscr{N}]$, and $Z_{p}, p=1, \ldots, k$ is $a$ basis of $[\mathcal{N}, \mathscr{N}]$. Then the operator

$$
D=X_{1}^{2}+X_{2}^{2} \text {, }
$$

where $X_{1}=\alpha_{1} Y_{1}+\cdots+\alpha_{n} Y_{n}, X_{2}=\beta_{1} Z_{1}+\cdots+\beta_{k} Z_{k}$, is $(G H)$ on the compact nilmanifold $\Gamma \backslash N$, provided both $\alpha_{1}, \ldots, \alpha_{n}$ and $\beta_{1}, \ldots, \beta_{k}$ satisfy the following growth condition (which we state for the $\alpha$ 's only):

$$
\left|\alpha_{1} k_{1}+\cdots+\alpha_{n} k_{n}\right| \geq C\left(k_{1}^{2}+\cdots+k_{n}^{2}\right)^{-p},
$$

for some $p, C>0$ and all integers $k_{1}, \ldots, k_{n}$ not all zero.

Proof. Let $D u=g \in C^{\infty}(\Gamma \backslash N)$ with $u \in \mathscr{D}^{\prime}(\Gamma \backslash N)$. We use an irreducible (non-canonical) Fourier series decomposition of $u$, $u=u_{0}+\sum_{\pi} \sum_{q=1}^{m(\pi)} u_{\pi, q}$, where $u_{0} \in \mathscr{D}^{\prime}(\Gamma[N, N] \backslash N)$. Thus $u_{0}$ lives on the associated torus. The sum is over all $\infty$-dimensional representations $\pi \in(\Gamma \backslash N)^{\wedge}$ (with multiplicities $m(\pi)$ ). Also, $g$ has a Fourier series decomposition with $g_{0} \in C^{\infty}(\Gamma[N, N] \backslash N)$. Condition (1.2) assures that the operator $\bar{D}=\left(\alpha_{1} \partial / \partial x_{1}+\cdots+\alpha_{n} \partial / \partial x_{n}\right)^{2}$ on the associated torus is $(\mathrm{GH})$ by the Theorem in [G-W1]. We conclude that $u_{0}$ is in fact smooth. The proof that the sum over $\infty$-dimensional $\pi$ is smooth is a modification of the proof of global regularity of a real vector field on a compact nilmanifold (Theorem 1, page 351 of [C-R3]). For each fixed $\infty$-dimensional $\pi$ we construct a suitable Schrödinger model. Since $\pi$ is $\infty$-dimensional, there exists $i$ such that $\left[Y_{i}, X_{1}\right] \notin \operatorname{ker}(d \pi)$. Let $\mathscr{W}_{\pi}$ be an ideal in $\operatorname{ker}(d \pi)$ such that $\mathscr{N} / \mathscr{W}_{\pi}$ has 1 -dimensional center. Passage to this quotient does not affect $d \pi(D)$. Introducing a Kirillov subalgebra generated by the images 
of $X:=Y_{i}$ and $Y:=X_{1}$ in $\mathscr{N} / \mathscr{W}_{\pi}$, we obtain a Schrödinger model for $\pi$. In that model $d \pi(D)=-\lambda^{2} \xi_{1}^{2}-\Lambda\left(X_{2}\right)^{2}$, where $\Lambda \in \mathscr{N}^{*}$ corresponds via Kirillov theory $[\mathbf{K}]$ to $\pi$. Moreover, $\Lambda([\mathscr{N}, \mathscr{N}] \cap \log \Gamma) \subseteq \mathbb{Z}$ and $\lambda=\Lambda\left(\left[Y_{1}, X_{1}\right]\right)$. We use the formula (1.8) on page 353 of [C-R1] to write for any $U \in \mathscr{U}(\mathcal{N})$, the universal enveloping algebra of $\mathscr{N}$ :

$$
\begin{aligned}
(U f)_{\pi}= & \pi\{[D[D \ldots[D, U] \ldots]] g+D[D \ldots[D, U] \ldots] g \\
& \left.+\cdots+D^{m-2}[D, U] g+D^{m-1} U g\right\} P_{\pi}^{-m} \\
& \equiv h_{m} P_{\pi}^{-m} .
\end{aligned}
$$

Here $P_{\pi}\left(\xi_{1}, \ldots, \xi_{k}\right)=-\lambda^{2} \xi_{1}^{2}-\Lambda\left(X_{2}\right)^{2}$ and (instead of (1.9) on page 353 of $[\mathbf{C}-\mathbf{R 1}])$ we use the estimate

$$
\begin{aligned}
\left|h_{m} P_{\pi}^{-m}\right| & \leq\left|h_{m}\right|\left|\Lambda\left(X_{2}\right)\right|^{-2 m} \\
& \leq C^{-2}\left|\Lambda\left(Z_{1}\right)^{2}+\cdots+\Lambda\left(Z_{k}\right)^{2}\right|^{p}\left|h_{m}\right| \equiv\left|\pi(V) h_{m}\right|
\end{aligned}
$$

for some $V \in \mathscr{U}(\mathscr{N})$.

The second inequality is where we need the assumption (1.2) about the coefficients $\beta_{1}, \ldots, \beta_{k}$ of $X_{2}$. Also, (1.3) works only if $(\operatorname{ad} D)^{m} U$ $=0$ for some $m$ (depending of course on $U$ ). Since $D=X_{1}^{2}+X_{2}^{2}$ with $X_{2}$ central in $\mathscr{N}$, this is the same as $\left(\operatorname{ad}\left(X_{1}^{2}\right)\right)^{m} U=0$. The latter condition is true for any nilpotent Lie algebra $\mathscr{N}$, any $X_{1} \in \mathscr{N}$ and $U \in \mathscr{U}(\mathscr{N})$. To see this, wlog we assume that $U=U_{1} U_{2} \cdots U_{p}$ with $U_{i} \in \mathscr{N}, i=1, \ldots, p$. Note that $\operatorname{ad}\left(X_{1}^{2}\right)$ is a derivation of the associative algebra $\mathscr{U}(\mathscr{N})$. By Leibnitz's rule $\operatorname{ad}\left(X_{1}^{2}\right)^{m} U=a$ linear combination of the terms of the form of $\operatorname{ad}\left(X_{1}^{2}\right)^{l_{1}} U_{1} \cdots \operatorname{ad}\left(X_{1}^{2}\right)^{l_{p}} U_{p}$, where $l_{1}+\cdots+l_{p}=m$. Thus it suffices to show that there exists a number $l$ such that $\operatorname{ad}\left(X_{1}^{2}\right)^{l}$ maps $\mathscr{N}$ into 0 . This last statement is contained in Lemma 5.1 on page 230 of [G].

(1.4) ExAmple. Let $N$ be a direct product of the 3 dimensional Heisenberg group and $\mathbb{R}$. Let $X, Y, Z$, and $Z_{1}$ with $[X, Y]=Z$ be a rational basis of $\mathscr{N}$. Consider $D=(X+\alpha Y)^{2}+\left(Z+\beta Z_{1}\right)^{2}$ with $\alpha$ irrational non-Liouville and $\beta$ a Liouville number. As in the proof of Theorem (1.1), for $\pi \in(\Gamma \backslash N)^{\wedge}$, pick a Schrödinger model with Kirillov subalgebra generated by $Y$ and $X+\alpha Y$. In that model $d \pi(D)=-\lambda^{2} \xi^{2}-\left(\lambda+\beta \lambda_{1}^{2}\right)$ with $\lambda=\Lambda(Z), \lambda_{1}=\Lambda\left(Z_{1}\right)$, where $\Lambda \in \mathscr{N}^{*}$ corresponds to $\pi$. Computations similar to those of Example 1 on page 355 of [C-R3] show that $D$ cannot be $(\mathrm{GH})$ on $\Gamma \backslash N$.

2. $D$-harmonic distributions on nilmanifolds. The following Theorem (2.1) does not require any growth assumptions on $X_{1}, \ldots, X_{k}$. (Whether $D$ in the Theorem is $(\mathrm{GH})$, even with $X_{1}, \ldots, X_{k}$ and 
their commutators satisfying (1.2), is still an open problem. This problem is still open even if $X_{1}, \ldots, X_{k}$ are algebraic.) Consider the case $k=1$, with $\Gamma \backslash N$ being the torus, say two dimensional, and $D=\left(\alpha_{1} Y_{1}+\alpha_{2} Y_{2}\right)^{2}=X_{1}^{2}$. Then Theorem (1.1) corresponds to the statement that $D$ is $(\mathrm{GH})$ provided $\alpha_{2} / \alpha_{1}$ is an irrational nonLiouville number. On the other hand, the 2-torus version of Theorem (2.1) says that $\operatorname{ker} D=\mathbb{C} \cdot 1$ provided $\alpha_{2} / \alpha_{1}$ is irrational.

(2.1) Theorem. Let $\mathcal{N}$ be a rational nilpotent Lie algebra of step $r, N$ the corresponding connected, simply connected group, and $\Gamma$ a cocompact discrete subgroup of $N$. Let $X_{1}, \ldots, X_{k}$ generate a Lie subalgebra $\mathscr{L}$ of $\mathscr{N}$. Suppose that $\mathscr{L}$ has the property

(2.2) For each non-zero integral linear functional $\Lambda \in\left(\mathscr{N}_{j} / \mathscr{N}_{j+1}\right)^{*}$, $\Lambda\left(\left(\mathscr{L} \cap \mathscr{N}_{j}\right)+\mathscr{N}_{j+1}\right) \neq 0, j=1, \ldots, r$.

$\left[\Lambda \in \mathscr{N}_{j}^{*}\right.$ is called integral if $\left.\Lambda\left(\log \Gamma \cap \mathscr{N}_{j}\right) \subset \mathbb{Z}.\right]$

If $u \in \mathscr{D}^{\prime}(\Gamma \backslash N)$ and $\left(X_{1}^{2}+\cdots+X_{k}^{2}\right) u=0$, then $u$ can be identified with a constant function.

REMARK 1. $X_{1}, \ldots, X_{k}$ satisfying condition (2.2) in general do not generate the whole tangent space of $\Gamma \backslash N$. Consequently, $D$-harmonic distributions a priori need not even be continuous functions. Therefore, compactness of $\Gamma \backslash N$ alone cannot guarantee such 'harmonic' distributions to be constants.

REMARK 2. If $r=1$, then Theorem (2.2) is about a torus. (Recall that $\mathscr{N}=\mathscr{N}_{1}$ and $\mathscr{N}_{2}$ is the commutator of $\mathscr{N}$.)

We start the proof of Theorem (2.1) with the following proposition.

(2.3) Proposition. The condition (2.2) above and the following condition (2.4) are equivalent for every compact nilmanifold $\Gamma \backslash N$.

(2.4) For each $\pi \in(\Gamma \backslash N)^{\wedge} \sim\{1\}$, if $1 \leq j \leq r$ is such that $\pi\left(N_{j+1}\right) \equiv I$, but $\pi\left(N_{j}\right) \not \equiv I$, then $d \pi\left(\mathscr{L} \cap \mathscr{N}_{j}\right) \neq 0$, where $N_{j}=\exp \mathscr{N}_{j}$, and $(\Gamma \backslash N)^{\wedge}$ denotes the irreducible unitary representations of $N$ contained in the quasiregular representation of $N$ on $L^{2}(\Gamma \backslash N)$.

Proof $(2.2) \Leftrightarrow(2.4)$. Each $\pi \in(\Gamma \backslash N)^{\wedge}$ corresponds to some $\tilde{\Lambda} \in$ $\mathscr{N}^{*}$ integral on a rational maximal subordinate subalgebra $\mathscr{M}$ of $\mathscr{N}$ 
([H],[R1]). In particular, for $j$ as in (2.4) $\mathscr{N}_{j} \subseteq \mathscr{M}$, and

$$
\left.d \pi\right|_{\mathscr{N}_{j}}=\left.i \widetilde{\Lambda}\right|_{\mathscr{N}_{j}}=i \Lambda \quad \text { with } \Lambda \in\left(\mathscr{N}_{j} / \mathscr{N}_{j+1}\right)^{*} \text { integral. }
$$

Conversely, any integral $\Lambda \in\left(\mathscr{N}_{j} / \mathscr{N}_{j+1}\right)^{*}$ can be extended by 0 on a rational basis of $\mathscr{N}$ to an integral $\widetilde{\Lambda} \in \mathscr{N}^{*} . \pi \in \widehat{N}$ corresponding via Kirillov theory to $\widetilde{\Lambda}$ is in the spectrum of $\Gamma \backslash N$ ([M]). Equation (2.5) holds as before. Thus (2.2) and (2.4) are equivalent.

In view of the Proposition (2.3), all we need to prove Theorem (2.1) is the following:

(2.6) Lemma. Let $X_{1}, \ldots, X_{k}$ generate a Lie subalgebra $\mathscr{L}$ of a nilpotent Lie algebra $\mathscr{N}$. Let $\pi \in \widehat{N}$ be such that $d \pi\left(\mathscr{L} \cap \mathscr{N}_{r}\right) \neq 0$. Then for every $u_{\pi} \in\left(H_{\pi}^{\infty}\right)^{\prime}, d \pi\left(X_{1}^{2}+\cdots+X_{k}^{2}\right) u_{\pi}=0$ implies $u_{\pi}=0$. Here $N=\exp \mathscr{N}$ and $\mathscr{N}_{r}$ is the lowest non-zero term of the lower central series of $\mathscr{N}$.

Proof of Theorem (2.1). We write an irreducible Fourier series expansion

$$
u=\sum_{\pi \in(\Gamma \backslash N)^{\wedge}} \sum_{q=1}^{m_{\pi}} u_{\pi, q}=u_{0}+\sum_{j=1}^{r} \sum_{\pi \in \Pi,} \sum_{q=1}^{m_{\pi}} u_{\pi, q},
$$

where

$$
\Pi_{j}=\left\{\pi \in(\Gamma \backslash N)^{\wedge}: \pi\left(N_{j+1}\right) \equiv I, \pi\left(N_{j}\right) \not \equiv I\right\}, \quad j=1, \ldots, r .
$$

Note that $\Pi_{1}$ consists of all 1-dimensional non-trivial representations in $(\Gamma \backslash N)^{\wedge}$. We apply Lemma (2.6) to $u_{\pi, q}$ with $\pi \in \Pi_{r}$, then again apply Lemma (2.6) to $\mathscr{N} / \mathscr{N}_{r}$ which takes care of $u_{\pi, q}$ with $\pi \in \Pi_{r-1}$ in the above sum, etc. We are left with $u_{0}$ which corresponds to trivial $\pi$, i.e. $u=u_{0}$ is a constant function on $M$.

The proof of Lemma (2.6) will follow from Lemma (2.7) below, but first we need some definitions (cf. [F-S]).

A Lie algebra $\mathscr{L}$ is called graded if it has a direct sum decomposition $\mathscr{L}=\sum_{j=1}^{r} \oplus V^{j}$ with the property that $\left[V^{j}, V^{k}\right] \subset V^{k+j}$ if $k+j \leq r$ and $\left[V^{k}, V^{j}\right]=0$ if $k+j>r$. A graded algebra is always nilpotent. A connected simply connected nilpotent Lie group $L$ is called graded if its Lie algebra $\mathscr{L}$ is graded.

Any graded (nilpotent) Lie algebra $\mathscr{L}$ has a natural family of dilations $\left\{\alpha_{\lambda}\right\}_{\lambda>0}$ (one parameter group of automorphisms of $\mathscr{L}$ ) defined on each $V^{j}$ by $\alpha_{j}(Y)=\lambda^{j} Y, Y \in V^{j}, \lambda>0$. By the exponential 
map $\alpha_{\lambda}$ corresponds to a one-parameter group of automorphisms of $L$, the simply connected nilpotent Lie group corresponding to $\mathscr{L}$.

A linear differential operator $P$ on a graded group $L$ is homogeneous of degree $d$ if $P\left(f \circ \alpha_{\lambda}\right)=\lambda^{d}(P f) \circ \alpha_{\lambda}$ for any $f \in C^{\infty}(L)$.

We call a differential operator $P$ on a graded group $L$ a Rockland operator if (i) $P$ is left-invariant and homogeneous, and (ii) $d \pi(P)$ is injective on $H_{\pi}^{\infty}$ for every $\pi \in \widehat{L}$ except the trivial representation. By a theorem of Helffer and Nourrigat [H-N], a Rockland operator (on a graded group $L$ ) is hypoelliptic: i.e. if $u$ is a distribution on $L$ such that $P u$ is $C^{\infty}$ on an open $\Omega \subset L$, then $u$ is $C^{\infty}$ on $\Omega$.

(2.7) LemMA. Let $\mathscr{L}$ be a graded Lie subalgebra of a nilpotent Lie algebra $\mathscr{N}$, and let $P \in \mathscr{U}(\mathscr{L})$, the universal enveloping algebra of $\mathscr{L}$, be a Rockland operator on the graded group $L$ corresponding to $\mathscr{L}$. If $\pi \in \widehat{N}$ is such that $d \pi\left(\mathscr{L} \cap \mathscr{N}_{r}\right) \neq 0$, then $d \pi(P) u_{\pi}=0$ for $u_{\pi} \in\left(H_{\pi}^{\infty}\right)^{\prime}$ implies $u_{\pi}=0$. ( $\mathscr{N}_{r}$ is the lowest non-zero term of the lower central series of $\mathscr{N}$.)

Proof of Lemma (2.7). Suppose $d \pi(P) u=0$ for some $0 \neq u \in$ $\left(H_{\pi}^{\infty}\right)^{\prime}$. We are going to show then there is a non-smooth function $\tilde{u}$ on $L$ such that $P \tilde{u}=0$. That would contradict the hypoellipticity of $P$ on $L$. We adapt the proof of Lemma (4.6) of Rothschild and Stein [R-S] to our situation. Let $\psi \in H_{\pi}^{\infty}$ be such that $(u, \psi) \neq 0$, and let $\left\{\alpha_{\lambda}\right\}_{\lambda>0}$ be the one-parameter group of dilations of $L$. For each dilation $l \rightarrow \alpha_{\lambda}(l)\left(\lambda \in \mathbb{R}^{+}\right)$of $L$ define the representation $\pi_{\lambda}$ of $L$ by $\pi_{\lambda}(l):=\pi\left(\alpha_{\lambda}(l)\right)$. Observe that if $\pi(P) u=0$, it follows from the homogeneity of $P$ that $\pi_{\lambda}(P) u=0$ too. Let

$$
\tilde{u}(l)=\int_{1}^{\infty}\left(\pi_{\lambda}(l) u, \psi\right) \lambda^{Q} d \lambda, \quad l \in L,
$$

where the exponent $Q$ is to be specified later. First we check that the integral in (2.8) converges for each fixed $l \in L$. Since $u \in\left(H_{\pi}^{\infty}\right)^{\prime}$, we have

$$
\left|\left(\pi_{\lambda}(l) u, \psi\right)\right|=\left|\left(u, \pi\left(\alpha_{\lambda}\left(l^{-1}\right)\right) \psi\right)\right| \leq C||\left|\pi\left(\alpha_{\lambda}\left(l^{-1}\right)\right) \psi\right||| .
$$

By $[\mathbf{K}], H_{\pi}$ can be identified with $L^{2}\left(\mathbb{R}^{P}\right), H_{\pi}^{\infty}$ with $\mathscr{S}\left(\mathbb{R}^{P}\right)$, the Schwartz space of rapidly decreasing functions, and we can think of $\||\cdot|||$ in (2.9) as being a combination of $\mathscr{S}\left(\mathbb{R}^{P}\right)$ seminorms of the form \|\|$\phi\|=\| x^{\beta} D_{\alpha} \phi(x) \|$, where \|\| is the $L^{2}\left(\mathbb{R}^{P}\right)$ norm. In our 
case

$$
\phi=\pi\left(\alpha_{\lambda}\left(l^{-1}\right)\right) \psi=\pi\left(\exp \left(\sum_{j} \lambda^{j} Y_{j}\right)\right) \psi
$$

where

$$
\log l=Y=\sum Y_{j} \in \bigoplus_{j=1}^{r} V^{j}=\mathscr{L} .
$$

The representation $\pi \in \widehat{N}$ acting on $\phi \in L^{2}\left(\mathbb{R}^{P}\right)$ can be written (cf. [H-N], page 904)

$$
\pi(\exp Y) \phi(x)=\exp (i\langle\Lambda, v(Y, x)\rangle) \phi(\sigma(x, Y)),
$$

where $\sigma \in \mathbb{R}^{P} \quad(=\mathscr{M} \backslash \mathscr{N})$ and $v \in \mathscr{N}$ are polynomials in $x \in \mathbb{R}^{P}$ and $Y \in \mathscr{L}$, and $\mathscr{M} \subset \mathscr{N}$ is a maximal subordinate subalgebra for $\Lambda \in \mathscr{N}^{*}$. Combining (2.10) and (2.12) we see that $\|\phi\| \|$ can be estimated by a combination of expressions of the form

$$
\left\|v_{1}(\cdot, Y)\left(D_{\alpha} \psi\right)(\sigma(\cdot, Y))\right\|, \quad Y=\log l
$$

with $\sigma$ as in (2.12) and some polynomial $v_{1}$ of $x \in \mathbb{R}^{P}$ and $Y \in \mathscr{L}$, the norm \|\| being the $L^{2}\left(\mathbb{R}^{P}\right)$ norm with respect to the $x \in \mathbb{R}^{P}$ variable marked by a dot. Since $\pi$ is unitary, we can rewrite (2.13) as

$$
\left\|\pi(\exp (-Y))\left\{v_{1}(\cdot, Y)\left(D_{\alpha} \psi\right)(\sigma(\cdot, Y))\right\}\right\|
$$

which can be written

$$
\left\|v_{2}(\cdot, Y)\left(D_{\alpha} \psi\right)(\cdot)\right\|
$$

with some polynomial $v_{2}$. This is because $\sigma(x, Y)$ (see (2.12)) comes from the multiplication of $\exp X$ (on the right) by $\exp Y$, and subsequent multiplication of $\exp \sigma(x, Y)$ by $\exp (-Y)$ makes it $x$ again. Suppose now that $Y$ depends on $\lambda$ via $Y=Y_{\lambda}=\sum \lambda^{j} Y_{j}$ (cf. (2.11)). It follows from (2.13b) that

$\|\phi\| \mid \leq$ a polynomial in $\lambda$ of some degree $Q_{1}$ with coefficients of the form $\left\|v_{3}\left(\cdot ; Y_{1}, \ldots, Y_{r}\right)\left(D_{\alpha} \psi\right)(\cdot)\right\|$, $\left(v_{3}\right.$ being a polynomial in $x \in \mathbb{R}^{P}$ and $\left.Y_{1}, \ldots, Y_{r}\right)$ and with $Q_{1}$ independent of $Y$ or $x$.

Thus $\tilde{u}(l)$ is well-defined and continuous for any $Q \geq Q_{1}+2$. As in [R-S] $P \tilde{u}=0$, since the differentiation under the integral sign can 
be justified as follows. (Recall that $P$ acts on the right and is leftinvariant.)

$$
\begin{aligned}
& \left|P\left(\pi_{\lambda}(l) u, \psi\right)\right|=\left|\left(\pi_{\lambda}(l) \pi_{\lambda}(P) u, \psi\right)\right| \\
& \quad=\lambda^{d}\left|\left(\pi_{\lambda}(l) \pi(P) u, \psi\right)\right|=\lambda^{d}\left|\left(u, \pi\left({ }^{t} P\right) \pi\left(l^{-1}\right) \psi\right)\right| \\
& \quad \leq \lambda^{d} C||\left|\pi\left({ }^{t} P\right) \pi\left(\alpha_{\lambda}\left(l^{-1}\right)\right) \psi\right||| \leq\left.\lambda^{d} C_{1}||\left|\pi\left(\alpha_{\lambda}\left(l^{-1}\right) \psi\right)\right|\right|^{\prime}
\end{aligned}
$$

where $d=$ degree of homogeneity of $P$ and $\|||||^{\prime}$ means that we've "absorbed" $\pi(P)$ into the Schwartz space seminorm ||| |||. The last expression in (2.15) can now be estimated in exactly the same way as the one in (2.9), resulting in an estimate similar to (2.14), with a polynomial in $\lambda$ of degree $Q_{2}$, say. Thus $\int_{1}^{\infty} P\left(\pi_{\lambda}(l) u, \psi\right) \lambda-Q d \lambda$ converges absolutely whenever $Q \geq Q_{2}+2$, and $P \tilde{u}=\int_{1}^{\infty} P\left(\pi_{\lambda}(l) u, \psi\right) \lambda-Q d \lambda=$ $\int_{1}^{\infty}\left(\pi_{\lambda}(l) \pi_{\lambda}(P) u, \psi\right) \lambda-Q d \lambda=0$.

The key thing now is that by the assumption of the lemma there is a $Z \in \mathscr{N}_{r} \cap \mathscr{L}$ such that $d \pi(Z)=i c \neq 0, c \in \mathbb{R}$. For this $Z$ we have $\pi_{\lambda}(\exp t Z)=e^{i c t \lambda^{r}}$ and

$$
\begin{aligned}
\tilde{u}(\exp t Z) & =\int_{1}^{\infty}(\pi(\exp t Z) u, \psi) \lambda^{-Q} d \lambda \\
& =(u, \psi) \int_{1}^{\infty} e^{i c t \lambda^{r}} \lambda^{-Q} d \lambda \\
& =(u, \psi) r^{-1} \int_{1}^{\infty} e^{i c t \lambda^{\prime}} \lambda^{\prime-Q_{3}} d \lambda^{\prime}
\end{aligned}
$$

where $Q_{3}=Q / r+(r-1) / r$ and $\lambda^{\prime}=\lambda^{r}$. We pick now $Q$ in (2.8) so that $Q \geq \max \left(Q_{1}, Q_{2}\right)+2$, and that $Q_{3}$ is an integer $\geq 2$. With this choice of $Q$, as in [R-S] $\tilde{u}(l)$ defines a distribution on $L, P \tilde{u} \equiv 0$, yet $\tilde{u}$ restricted to $\exp (\mathbb{R} Z) \subset L$ is not smooth.

(2.16) COROllaRY. In particular, Lemma (2.7) holds true for $\mathscr{N}=$ $\mathscr{F}$, the free nilpotent Lie algebra of step $r$ on $k+m$ generators $\widetilde{X}_{1}, \ldots, \widetilde{X}_{k} ; \widetilde{Y}_{1}, \ldots, \widetilde{Y}_{m}$, and $\widetilde{\mathscr{L}}$ the subalgebra of $\mathscr{F}$ generated by $\tilde{X}_{1}, \ldots, \widetilde{X}_{k}$. Here $P=\widetilde{X}_{1}^{2}+\cdots+\widetilde{X}_{k}^{2}$ is the Rockland operator (cf. [F-S], p. 130) and $\rho \in \widehat{F}$ is such that $d \rho(\widetilde{Z}) \neq 0$ for some $\widetilde{Z} \in \widetilde{L} \cap \mathscr{F}_{r}$.

Proof of Lemma (2.6). Suppose $d \pi\left(X_{1}^{2}+\cdots+X_{k}^{2}\right) u=0$ for $u \in$ $\left(H_{\pi}^{\infty}\right)^{\prime}$ and let $Z \in \mathscr{L} \cap \mathscr{N}_{r}$ be such that $d \pi(Z) \neq 0$. By the above corollary, $u=0$. This can be seen as follows. We construct a chain of subgroups from $L$ to $N$, each of codimension 1 in the next, so that $\mathscr{N}=\mathbb{R} Y_{1} \ltimes \cdots \ltimes \mathbb{R} Y_{m} \ltimes \mathscr{L}$, for some $Y_{1}, \ldots, Y_{m} \in \mathscr{N}$. Let $\Phi$ be a homomorphism of $\mathscr{F}$ onto $\mathscr{N}$ given on the generators of $\mathscr{F}$ by 
$\Phi\left(\widetilde{X}_{j}\right)=X_{j}, j=1, \ldots, k ; \Phi\left(\widetilde{Y}_{j}\right)=Y_{j}, j=1, \ldots, m$. Let $\tilde{Z}$ be a preimage of $Z$ in $\widetilde{\mathscr{L}} \cap \mathscr{F}_{r}$. We apply the corollary to $\rho=\pi \circ \Phi \in \widehat{F}$ noticing that $H_{\pi}=H_{\rho}$ and $d \rho\left(\widetilde{X}_{1}^{2}+\cdots+\widetilde{X}_{k}^{2}\right)=d \pi\left(X_{1}^{2}+\cdots+X_{k}^{2}\right)$.

Lemma (2.7) also implies the following version of Theorem (2.1) in case $\mathscr{L}$, the Lie algebra generated by $X_{1}, \ldots, X_{k}$, is graded.

(2.1') Theorem. Let $M=\Gamma \backslash N$ be a compact nilmanifold and let $\mathscr{L}$ be a graded subalgebra of $\mathscr{N}$. Suppose that $\mathscr{L}$ has the property (2.2). Let $P \in \mathscr{U}(\mathscr{L})$ be a Rockland operator on $L$ acting on $\Gamma \backslash N$. If $u \in \mathscr{D}^{\prime}(\Gamma \backslash N)$ is in the kernel of $P$ then $u=$ const.

Remark 1. Theorem $\left(2.1^{\prime}\right)$ states that if $\mathscr{L}$ is a large enough graded subalgebra of $\mathscr{N}$ (i.e. $\mathscr{L}$ satisfies (2.2)) and $P \in \mathscr{U}(\mathscr{L})$ is homogeneous, then injectivity of $d \rho(P)$ on $H_{\rho}^{\infty}$ for all non-trivial $\rho$ in $\widehat{L}$ implies injectivity of $d \pi(P)$ on $\left(H_{\pi}^{\infty}\right)^{\prime}$ for all non-trivial $\pi$ in $(\Gamma \backslash N)^{\wedge}$.

REMARK 2. The existence of $Z$ in $\mathscr{N}_{r} \cap \mathscr{L}$ at the end of the proof of Lemma (2.7) and the choice of $\widetilde{Z}$ in $\widetilde{\mathscr{L}} \cap \mathscr{F}_{r}$ in the proof of Lemma (2.6) use condition (2.2). We don't know whether the assumption (2.2) in Theorem (2.1) can be replaced by the weaker condition $\left(2^{\circ}\right)$ of Theorem (4.1).

3. A solvmanifold (counter)example. Here we produce an example of a $(\mathrm{GH})$ system of two vector fields on a class of 3-dimensional ("hyperbolic") solvmanifolds. We show that the kernel of the sum of squares of these vector fields contains a distribution which is not a $C^{\infty}$-function. Also, Lemma (3.4) might be of independent interest.

Consider the following class of three-dimensional compact solvmanifolds, $M=\Gamma \backslash S$ (see [A-G-H] and [Br1, 2] for details). $S$ is the semidirect product of $\mathbb{R}$ and $\mathbb{R}^{2}$ (with $\mathbb{R}^{2}$ normal in $S$ ), in which the group operation is

$$
(x, t)\left(x^{\prime}, t^{\prime}\right)=\left(x+A^{t} x^{\prime}, t+t^{\prime}\right), \quad x, x^{\prime} \in \mathbb{R}^{2}, t, t^{\prime} \in \mathbb{R} .
$$

Here $A^{t}, t \in \mathbb{R}$, is a 1-parameter subgroup of $\operatorname{SL}(2, \mathbb{R})$ through a fixed matrix $A \in \operatorname{SL}(2, \mathbb{Z})$. The discrete subgroup $\Gamma$ can be taken to be the set of points in $S$ with integer coordinates. (The fact that $A \in$ $\mathrm{SL}(2, \mathbb{Z})$ is equivalent to $A$ mapping the integer lattice $\mathbb{Z}^{2}$ into itself.) We'll consider the case in which $A$ has unequal positive eigenvalues $\lambda$ and $\lambda^{-1}$. Choosing the eigenvectors of $A$ as a basis of $\mathbb{R}^{2}$ we can 
write the group operation in $S$ in the new $u, v$ coordinates

$$
\begin{array}{r}
(u, v, t)\left(u^{\prime}, v^{\prime}, t^{\prime}\right)=\left(u+\lambda^{t} u^{\prime}, v+\lambda^{-t} v^{\prime}, t+t^{\prime}\right), \\
u, u^{\prime}, v, v^{\prime}, t, t^{\prime} \in \mathbb{R} .
\end{array}
$$

In these new coordinates $\mathbb{R}^{2} \cap \Gamma$ is no longer $\mathbb{Z}^{2}$. (For each $\lambda>1$ such that $\lambda, \lambda^{-1}$ are eigenvalues of a matrix $A \in \mathrm{SL}(2, \mathbb{Z})$ we get a distinct solvmanifold $\Gamma_{\lambda} \backslash S$, although $S$ is not changed up to isomorphism by altering $\lambda$.) Letting $T, U$, and $V$ be the infinitesimal generators of the one-parameter subgroups of $S$ corresponding to $t, u$, and $v$ we have $[T, U]=\ln \lambda U,[T, V]=-\ln \lambda V$. We consider the operator $P=T^{2}+U^{2}$ and the system $\{T, U\}$ of vector fields induced on $\Gamma \backslash S$ by $T$ and $U$.

(3.2) Proposition. Let $T, U$ and $\Gamma \backslash S$ be as described above. Then

(a) The system of vector fields $\{T, U\}$ on $M=\Gamma \backslash S$ is $(G H)$;

(b) The operator $P=T^{2}+U^{2}$ is not $(G H)$ on $\Gamma \backslash S$. In fact, there is a distribution $u \in \mathscr{D}^{\prime}(\Gamma \backslash S) \sim L^{2}(\Gamma \backslash S)$ such that $P u=0$.

Proof of (a). Let $u \in \mathscr{D}^{\prime}(\Gamma \backslash S)$ be such that $T u=f, U u=g$, and $V u=h$, with $f, g, h \in C^{\infty}(\Gamma \backslash S)$. Let $u=u_{0}+\sum_{\pi, j} u_{\pi, j}$ be an irreducible Fourier series of $u$, the summation being over infinite dimensional $\pi \in(\Gamma \backslash S)^{-}$with $j$ counting the multiplicities and with $u_{0} \in \mathscr{D}^{\prime}(\Gamma[S, S] \backslash S)$, so $u_{0}$ lives on the associated torus. On that 1dim torus, $T$ acts as $d / d t$. Thus $T u_{0}=f_{0} \in C^{\infty}(\Gamma[S, S] \backslash S)$ and $u_{0}$ is smooth. As for the $u_{\pi, j}$ 's, each $\infty$-dimensional $\pi \in(\Gamma \backslash S)^{\wedge}$ acts on $L^{2}(\mathbb{R}, d t)$ and $d \pi(U) u_{\pi, j}=2 \pi i \alpha \lambda^{t} u_{\pi, j}$ for some $0 \neq \alpha \in \mathbb{R}$. Since $d \pi(V)$ acts by multiplication by $2 \pi i \beta \lambda^{-t}$ with $0 \neq \beta \in \mathbb{R}, u_{\pi, j}=$ $\left(-4 \pi^{2} \alpha \beta\right)^{-1} d \pi(V) d \pi(U) u_{\pi, j}=\left(-4 \pi^{2} \alpha \beta\right)^{-1}(V g)_{\pi, j}$. In fact, for any $R \in \mathscr{U}(\mathscr{S})$ we have $(R u)_{\pi, j}=\left(-4 \pi^{2} \alpha \beta\right)^{-1}(R V g)_{\pi, j}$, and

$$
\begin{aligned}
\|R u\|_{L^{2}(\Gamma \backslash S)}^{2} & =\left(4 \pi^{2}\right)^{-2} \sum_{\pi, j}\|R V g\|^{2}(\alpha \beta)^{-2}+\left\|(R u)_{0}\right\|^{2} \\
& \leq C \sum_{\pi, j}\|R V g\|^{2}+\left\|(R u)_{0}\right\|^{2} \\
& \leq C\|R V g\|_{L^{2}(\Gamma \backslash S)}^{2}<\infty .
\end{aligned}
$$

The last expression is finite since $g \in C^{\infty}(\Gamma \backslash S)$. The first inequality in (3.3) is a consequence of the following.

(3.4) LemmA. Let $S=\mathbb{R}^{2} \rtimes \mathbb{R}$ be a solvable Lie group with the group law (3.1). Let $\Gamma$ be a cocompact discrete subgroup of $S$. Then 
$\Gamma \cap \mathbb{R}^{2} \times\{0\}$ is an abelian lattice of points $(a, b) \in \mathbb{R}^{2}$ having the property that the product $a b$ is bounded away from zero, except of course for the group identity.

(3.5) COROLlARY. In the setting of the lemma above, the dual lattice $\Gamma^{*}=\left\{\chi_{\alpha, \beta}: \Gamma \rightarrow 1\right\}$ is also a lattice of points $(\alpha, \beta)$ such that the product $\alpha \beta$ is bounded away from 0 , except for $(\alpha, \beta)=(0,0)$.

Proof of Lemma (3.4). Let $(0,0, m)$ and $(a, b, 0) \in \Gamma \subset S$. Then $(0,0, m)(a, b, 0)(0,0, m)^{-1}=\left(\lambda^{m} a, \lambda^{-m} b, 0\right)$. Suppose $\left(a_{n}, b_{n}, 0\right), n=1,2, \ldots$ were a sequence of points in $\Gamma \cap \mathbb{R}^{2} \times\{0\}$ such that $a_{n} b_{n} \rightarrow 0$ as $n \rightarrow \infty$. Wlog we may suppose $a_{n} \geq b_{n}>0$. Then for every $n$ there would be an integer $k_{n}$ such that

$$
\lambda^{2\left(k_{n}-1\right)}<b_{n} / a_{n} \leq \lambda^{2 k_{n}} .
$$

Define a new sequence of points of $\Gamma$ by

$$
\left(a_{m}^{\prime}, b_{n}^{\prime}, 0\right):=\left(\lambda^{k_{n}} a_{n}, \lambda^{-k_{n}} b_{n}, 0\right), \quad n=1,2, \ldots
$$

We have $a_{n}^{\prime} b_{n}^{\prime}=a_{n} b_{n} \rightarrow 0$ and $b_{n}^{\prime} / a_{n}^{\prime}=\lambda^{-2 k_{n}} b_{n} / a_{n}$. The inequalities (3.6) imply now

$$
\lambda^{-2}<b_{n}^{\prime} / a^{\prime} \leq 1, \quad n=1,2, \ldots .
$$

Thus $\Gamma \ni\left(a_{n}^{\prime}, b_{n}^{\prime}, 0\right) \rightarrow(0,0,0)$ as $n \rightarrow \infty$, which violates the discreteness of $\Gamma$.

Proof of $(\mathrm{b})$. For a fixed infinite dimensional $\pi \in(\Gamma \backslash S)^{\wedge}$ acting on $L^{2}(\mathbb{R}, d t)$ we'll construct a non-zero function $u(t)$ on $\mathbb{R}$ such that $d \pi(P) u=0$ and $d \pi(U) u \in L^{2}(\mathbb{R})$. Such a $u$ defines a distribution $\tilde{u}$ on $\phi \in C^{\infty}(\Gamma \backslash S)$ :

$$
\begin{aligned}
|(\tilde{u}, \phi)| & :=\left|\left(u, Q \phi_{\pi}\right)\right| \\
& =\left|\left(u,\left(-4 \pi^{2} \alpha \beta\right)^{-1} \pi(U) \pi(V) Q \phi_{\pi}\right)\right| \\
& =\left|\left(\pi(U) u,(V Q \phi)_{\pi}\right)\right|\left|4 \pi^{2} \alpha \beta\right|^{-1} \\
& \leq C\|\pi(U) u\|\left\|(V \phi)_{\pi}\right\| \leq C_{1}\|V \phi\|_{L^{2}(\Gamma \backslash S)} \\
& \leq C_{2}\|V \phi\|_{L^{\infty}(\Gamma \backslash S)},
\end{aligned}
$$

where $\phi_{\pi}$ denotes a projection onto a fixed irreducible subspace $H_{\pi}$ of $L^{2}(\Gamma \backslash S)$ corresponding to $\pi$, and $Q: H_{\pi} \rightarrow L^{2}(\mathbb{R})$ is an intertwining operator onto a Schrödinger model for $\pi$.

To find such $u$ we write

$$
\begin{aligned}
d \pi(P) u & =\left(d^{2} / d t^{2}-4 \pi^{2} \alpha^{2} \lambda^{2 t}\right) u \\
& =\left\{(\ln \lambda) r^{2}\left(d / d r^{2}+r^{-1} d / d r\right)-(2 \pi \alpha r)^{2}\right\} u_{1}
\end{aligned}
$$


where we have put $r=\lambda^{t}$, and we have defined $u_{1}(r)=u(t), r>0$, and we take advantage of the fact that $d^{2} / d r^{2}+r^{-1} d / d r, r>0$, is the radial part of the Laplacian $\Delta$ on the plane. Thus (3.7) becomes equivalent to

$$
\left(\Delta-a^{2}\right) u_{2}=0, \quad a=2 \pi \alpha / \ln \lambda
$$

for a radial function $u_{2}$ on $\mathbb{R}^{2} \sim 0$. A solution $u_{2}$ of

$$
\left(\Delta-a^{2}\right) u_{2}=\delta_{0} \quad \text { on } \mathbb{R}^{2},
$$

where $\delta_{0}$ is the Dirac function supported at the origin 0 of $\mathbb{R}^{2}$, satisfies (3.8), and if it is radial, also (3.7). Applying the Fourier transform on $\mathbb{R}^{2}$ to (3.9) we obtain (cf. e.g. [S-W], page 6)

$$
\begin{aligned}
\hat{u}_{2}(\xi, \eta) & =-\left(4 \pi^{2}\right)^{-1}\left(\xi^{2}+\eta^{2}+a^{2}\right)^{-1} \\
& =-\left(4 \pi^{2}\right)^{-1} \int_{0}^{\infty} \exp \left[-\left(\xi^{2}+\eta^{2}+a^{2}\right) s\right] d s \\
& =\int_{\mathbb{R}^{2}} \exp [-2 \pi i(\xi x+\eta y)] u_{2}(x, y) d x d y
\end{aligned}
$$

where

(3.10) $u_{2}(x, y)=u_{1}(r)=-\left(16 \pi^{2}\right)^{-1} \int_{0}^{\infty} s^{-1} e^{-b s} \exp \left(-\pi^{2} r^{2} / s\right) d s$ with $r^{2}=x^{2}+y^{2}$ and $b=(\alpha / \ln \lambda)^{2}$. Thus letting $r=\lambda^{t},-\infty<$ $t<\infty, u_{1}(r)$ given by (3.10) is a non-zero solution to (3.7) we were after. It remains to show that $d \pi(U) u \in L^{2}(\mathbb{R})$. For this we write

$$
\begin{aligned}
\|d \pi(U) u\|^{2} & =\int_{-\infty}^{\infty}\left|2 \pi \alpha r u_{1}(r)\right|^{2} d t \\
& =c \int_{0}^{\infty} r\left(\int_{0}^{\infty} \ldots d s \int_{0}^{\infty} \ldots d \sigma\right) d r,
\end{aligned}
$$

where $c_{1} \int_{0}^{\infty} \ldots d s=u_{1}(r)=c_{1} \int_{0}^{\infty} \ldots d \sigma$ are given by (3.10). Changing the order of integration in (3.11) to $d r d s d \sigma$ and grouping the terms containing $r$ only, the $d r$ integral becomes

$$
\begin{aligned}
\int_{0}^{\infty} \exp \left[-\pi^{2} r^{2}\left(s^{-1}+\sigma^{-1}\right)\right] r d r & =\left(s^{-1}+\sigma^{-1}\right)^{-1} \int_{0}^{\infty} \exp \left(-\pi^{2} r^{2}\right) r d r \\
& =2 \pi^{2}\left(s^{-1}+\sigma^{-1}\right)^{-1}
\end{aligned}
$$


Substituting this back into (3.10) we obtain

$$
\begin{aligned}
(3.11) & =C^{\prime} \int_{0}^{\infty} \int_{0}^{\infty} \exp [-b(s+\sigma)](s \sigma)^{-1}\left(s^{-1}+\sigma^{-1}\right)^{-1} d s d \sigma \\
& =C^{\prime} \int_{0}^{\infty} \int_{0}^{\infty} \exp (-b s) \exp (-b \sigma)(s+\sigma)^{-1} d s d \sigma \\
& \leq\left(C^{\prime} / 2\right)\left(\int_{0}^{\infty} \exp (-b s) s^{-1 / 2} d s\right)^{2}<\infty
\end{aligned}
$$

REMARK. Similarly one can show that $\infty=\|u\|_{L^{2}(\mathbb{R})}$. We claim $\tilde{u} \in \mathscr{D}^{\prime}(\Gamma \backslash S)$ is not given by any $L^{2}$-function on $\Gamma \backslash S$. Suppose the negation, i.e. $\tilde{u}$ is given by some $w \in L^{2}(\Gamma \backslash S)$. Since $\tilde{u}: H_{\pi^{\prime}}^{\infty} \rightarrow 0$ for all $\pi^{\prime} \not \approx \pi, \pi^{\prime} \in(\Gamma \backslash S)^{\wedge}$, we have $w \in H_{\pi}$. Then $Q w \in L^{2}(\mathbb{R})$ and $Q w=u$ a.e. because it gives the same distribution-a contradiction. In particular, $\tilde{u}$ cannot be continuous or smooth on $\Gamma \backslash S$.

4. Necessary and sufficient conditions for (GH) of systems. Theorem 1 on page 366 of [C-R2] states a necessary and sufficient condition for $(\mathrm{GH})$ of a system $\mathbb{L}$ on $\Gamma \backslash N$. The proof of necessity, however, has a gap in the last paragraph of page 367. Namely, it is not clear whether or not there is a $\Lambda^{\prime} \in \mathscr{O}_{N}(\Lambda)$ such that $\Lambda^{\prime}(\mathscr{L})=0$. On the other hand, the proof of sufficiency establishes the (at least formally) stronger sufficiency theorem below.

(4.1) THEOREM. If $\left(1^{\circ}\right) \mathbb{L}+[\mathcal{N}, \mathscr{N}]$ is $(G H)$ on $\Gamma[N, N] \backslash N$, and $\left(2^{\circ}\right)$ for each $\pi \in(\Gamma \backslash N)_{\infty},\left(\mathscr{L}+\mathscr{W}_{\pi}\right) \cap \mathscr{Z}\left(\mathscr{N} / \mathscr{W}_{\pi}\right) \neq\{\overline{0}\}$, then $\mathbb{L}$ is $(G H)$ on $\Gamma \backslash N$. (Here, $\mathscr{W}_{\pi}$ is an ideal in $\operatorname{ker}(d \pi)$ such that $\operatorname{dim} \mathscr{Z}(\mathscr{N} / \mathscr{W})_{\pi}=1$ and $\pi \mid Z\left(N / W_{\pi}\right) \neq I$. Also, $\pi \in(\Gamma \backslash N)_{\infty}$ means $\pi$ is infinite dimensional, and $\mathscr{L}$ is a Lie subalgebra of $\mathscr{N}$ generated by $\mathbb{L}$.)

(4.2) Conjecture. Conditions $\left(1^{\circ}\right)$ and $\left(2^{\circ}\right)$ of Theorem (4.1) are necessary for $(\mathrm{GH})$ of $\mathscr{L}$ on $\Gamma \backslash N$.

Although we do not have any counter-example to this conjecture, we have been able to prove it only under special conditions.

To prove the conjecture, we assume that $\mathscr{L}$ is $(\mathrm{GH})$ on $\Gamma \backslash N$, so that $\left(1^{\circ}\right)$ is automatically satisfied. Then we suppose that $\bar{L} \cap$ $\mathscr{Z}(\mathscr{N} / \mathscr{W})_{\pi}=0$, and we try to prove there exists $\Lambda^{\prime} \in \mathscr{O}_{N}(\Lambda)$ such that $\Lambda^{\prime}(\mathscr{L})=0$. By the lemma on page 368 of [C-R2], this would contradict $(\mathrm{GH})$ of $\mathscr{L}$ on $\Gamma \backslash N$. 
(4.3) Proposition. Suppose that $\pi \in(\Gamma \backslash N)_{\infty}$ implies either that the corresponding co-adjoint orbit $\mathscr{O}(\pi)$ in $\mathscr{N}^{*}$ is flat, or else that $\pi$ is induceable from a polarization of codimension one in $\mathscr{N}$. If $\mathbb{L}=\left\{X_{1}, \ldots, X_{k}\right\}$ is a $(G H)$ system of vector fields on $\Gamma \backslash N$, then $\left(1^{\circ}\right)$ $\mathbb{L}+[\mathscr{N}, \mathscr{N}]$ is $(G H)$ on $\Gamma[N, N] \backslash N$, and $\left(2^{\circ}\right)$ for each $\pi \in(\Gamma \backslash N)_{\infty}$, $\left(\mathscr{L}+\mathscr{W}_{\pi}\right) \cap \mathscr{Z}\left(\mathscr{N} / \mathscr{W}_{\pi}\right) \neq\{\overline{0}\}$.

Before proving this theorem and giving examples, we state the following immediate consequences. If $\mathscr{N}$ is of step 2, then Proposition (4.3) shows that Conjecture (4.2) is true for $N$, since all orbits will be flat. Also, for each natural number $n \geq 2$, there exist nontrivial rational nilpotent Lie algebras of step $n$ such that all orbits (i.e., of all dimensions) will be flat [R3]. Thus the conjecture will have been proved for a large class of nilmanifolds. Also, the conjecture will have been proved for the important class of nilpotent semi-direct products $\mathbb{R} \ltimes \mathbb{R}^{n}$, with arbitrarily long lower central series.

Proof. The case of flat orbits is easiest. Fix a $\pi \in(\Gamma \backslash N)_{\infty}$. By repeatedly factoring out the part of the center in the kernel of $d \pi$, we may assume wlog that $\mathscr{Z}(\mathscr{N})=\mathbb{R} Z$, that $\mathscr{L} \cap \mathscr{Z}=\{0\}$, and that $\pi=\pi_{\Lambda}$ where $\Lambda=Z^{*}$. Then $\mathscr{O}_{N}(\Lambda)=Z^{*}+(Z)^{\perp} \cdot \mathscr{L}$ is spanned by a basis $L_{1}, \ldots, L_{k}$, not in $\mathscr{Z}$. Pick $\Lambda_{1}, \ldots, \Lambda_{k}$ in $Z^{\perp}$ such that

$$
\Lambda_{j}\left(L_{i}\right)= \begin{cases}0, & \text { if } i \neq j \\ -\Lambda\left(L_{j}\right), & \text { if } i=j .\end{cases}
$$

Then let $\Lambda^{\prime}=Z^{*}+\sum_{1}^{k} \Lambda_{j} \in \mathscr{O}_{N}(\Lambda)$, and $\Lambda^{\prime}(\mathscr{L})=0$. This proves the conjecture in the flat orbit case.

Now, suppose $\pi$ is induced from a (rational) polarization $\mathscr{M}$ of codimension 1. (Hence $\mathscr{M}$ is an ideal.)

(4.4) LemMA. If $\mathscr{Z}(\mathscr{N})=\mathbb{R} Z$ and $\mathscr{M}$ is a polarizing ideal for any $\Lambda \in \mathscr{N}^{*}$ with $\Lambda(Z) \neq 0$, then $\mathscr{M}$ is abelian.

Proof of Lemma. Since $\mathscr{M}$ is subordinate, $Z \notin[\mathscr{M}, \mathscr{M}]$, and $\mathscr{M} \triangleleft \mathcal{N}$ implies $[\mathscr{M}, \mathscr{M}]$ is an ideal too, since $(\operatorname{ad} X)\left[M_{1}, M_{2}\right]=\left[(\operatorname{ad} X) M_{1}\right.$, $\left.(\operatorname{ad} X) M_{2}\right]$. If there exists $0 \neq\left[M_{1}, M_{2}\right] \in[\mathscr{M}, \mathscr{M}]$, then $\left[M_{1}, M_{2}\right] \notin$ $\mathscr{Z}$, so there exists $U_{1} \in \mathcal{N}$ such that $\left(\operatorname{ad} U_{1}\right)\left[M_{1}, M_{2}\right]=\left[\left(\operatorname{ad} U_{1}\right) M_{1}\right.$, $\left.\left(\operatorname{ad} U_{1}\right) M_{2}\right] \in[\mathscr{M}, \mathscr{M}] \sim\{0\}$ too. Hence $\left(\operatorname{ad} U_{1}\right)\left[M_{1}, M_{2}\right] \in[\mathscr{M}, \mathscr{M}] \sim$ $\mathscr{Z}$. So there is a $U_{2}$ such that $\left[\left(\operatorname{ad} U_{2}\right)\left(\operatorname{ad} U_{1}\right) M_{1},\left(\operatorname{ad} U_{2}\right)\left(\operatorname{ad} U_{1}\right) M_{2}\right]$ $\neq 0$, and so on. Since there is no end to this process, the nilpotence of $\mathscr{N}$ has been violated. Thus $[\mathscr{M}, \mathscr{M}]=0$. 
This proves the lemma.

Now we have $\mathscr{N}=\mathbb{R} \ltimes \mathscr{M}$, where $\mathscr{M} \cong \mathbb{R}^{n}$ is a rational abelian ideal of codimension one in $\mathscr{N}$. Since $\mathscr{L}$ is $(\mathrm{GH})$ on $\Gamma \backslash N$, and since $\mathscr{M}$ is rational, $\mathscr{L} \not \subset \mathscr{M}$. Thus there exists $X \in \mathscr{L} \sim \mathscr{M}$. We are supposing $\mathscr{L} \cap \mathscr{Z}=\{0\}$, and it will suffice to prove that there exists $\Lambda^{\prime} \in \mathscr{O}_{N}(\Lambda)$ such that $\Lambda^{\prime}(\mathscr{L})=0$.

If $\mathscr{L}$ were not abelian, there would exist a central element $C \neq 0$, $C \in[\mathscr{N}, \mathscr{N}] \subset \mathscr{M}$, so $[C, \mathscr{M}]=0$. But $[C, X]=0$, and $\mathscr{N}=$ $\mathbb{R} X \oplus \mathscr{M}$. Thus $[C, \mathscr{N}]=0$, so that $C \in \mathscr{Z}(\mathscr{N}) \sim\{0\}$. But $C \in \mathscr{L}$, and $\mathscr{L} \cap \mathscr{Z} \neq\{0\}$. This is a contradiction, and so $\mathscr{L}$ is abelian.

Now, suppose there existed $L \in \mathscr{L} \cap \mathscr{M} \sim\{0\}$. Then $[X, L]=0=$ $[\mathscr{M}, L]$, so $L \in \mathscr{Z} \sim\{0\}$. This is a contradiction. So if $X \neq L \in$ $\mathscr{L} \sim\{0\}$, then $L=\alpha X+M$, for some $\alpha \neq 0$ and $M \in \mathscr{M}$. Also, since $\mathscr{L}$ is abelian, $[X, M]=0=[\mathscr{M}, M]$. Hence $M \in \mathscr{Z} \sim\{0\}$, so that $\mathscr{L}$ contains the $\mathbb{R}$-span of $X$ and $\alpha X+M$. But then $\mathscr{Z} \subset \mathscr{L}$. This is a contradiction.

Hence $\mathscr{L}=\mathbb{R} X$. Now, pick $Y \in \mathscr{M} \sim \mathscr{Z}$ such that $[X, Y]=Z$, and $\left(\operatorname{Ad}^{*} \exp \mathbb{R} Y\right) \Lambda=Z^{*}+\mathbb{R} X^{*}$. Hence there exists $\Lambda^{\prime} \in \mathscr{O}_{N}(\Lambda)$ such that $\Lambda^{\prime}(X)=0=\Lambda^{\prime}(\mathscr{L})$.

This proves the proposition.

(4.5) ExAmple. Let $\mathcal{N}$ be spanned by $X_{1}, X_{2}, Y_{1}, Y_{2}$, and $Z$, where all non-zero brackets are generated by $\left[X_{1}, Y_{1}\right]=Y_{2},\left[X_{1}, Y_{2}\right]$ $=Z$, and $\left[X_{2}, Y_{1}\right]=Z$. Thus $\mathscr{N}$ is 3-step, with $\mathscr{N}_{2}=[\mathcal{N}, \mathscr{N}]$ spanned by $Y_{2}$ and $Z$, and $\mathscr{Z}=\mathbb{R} Z$.

Let $X=X_{1}+\alpha Y_{1}$ and $Y=X_{2}+\alpha Y_{2}+\beta Z$, where $\alpha$ is an irrational, non-Liouville number. Then $\mathscr{L}=\mathbb{R} X \oplus \mathbb{R} Y$ is an abelian Lie subalgebra of $\mathscr{N},(\mathrm{GH})$ on $\Gamma[N, N] \backslash N$. Since $\mathscr{L} \cap \mathscr{Z}=\{0\}$, condition $\left(2^{\circ}\right)$ of Proposition (4.3) is not satisfied. However, every Kirillov orbit $\mathscr{O}_{N} \subset \mathscr{N}^{*}$ is flat (of all possible dimensions) [R3]. By Proposition (4.3), $\mathscr{L}$ is not $(\mathrm{GH})$ on $\Gamma \backslash N$, regardless of the choice of $\beta$, and regardless of the choice of $\alpha$. This example illustrates the necessity of conditions $\left(1^{\circ}\right)$ and $\left(2^{\circ}\right)$ of Proposition (4.3).

(4.6) EXAMPLE. Let $\mathcal{N}=\mathbb{R} \ltimes \mathbb{R}^{n+1}$ be the $n$-step nilpotent "chain" Lie algebra spanned by $X, Y_{1}, \ldots, Y_{n}, Y_{n+1}=Z$, with all nonzero brackets generated by $\left[X, Y_{i}\right]=Y_{i+1}, i=1, \ldots, n$. Then let $\mathscr{L}=\mathbb{R} L$, where $L=X+\alpha_{1} Y_{1}+\cdots+\alpha_{n} Y_{n}+\alpha_{n+1} Z$. Then $\mathscr{L}$ is (GH) on $\Gamma[N, N] \backslash N$, but $\mathscr{L}$ is not $(\mathrm{GH})$ on $\Gamma \backslash N$, since condition $\left(2^{\circ}\right)$ of Proposition (4.3) is not satisfied. That is $\mathscr{L} \cap \mathscr{Z}=\{0\}$. 
There are of course many variations and combinations of these two examples.

The following example supports Conjecture (4.2) by showing how $\mathscr{L} \cap \mathscr{Z}=\{0\}$ can lead to $\mathscr{L}$ not being $(\mathrm{GH})$ even under circumstances not covered by Proposition (4.3). In particular, it will be a 3-step nonflat orbit example in which $\mathscr{L}$ is $(\mathrm{GH})$ on $\Gamma[N, N] \backslash N$ and also on $\Gamma Z \backslash N$ and on $\Gamma M \backslash N$, and yet $\mathscr{L}$ is not (GH) on $\Gamma \backslash N$, apparently because $\mathscr{L} \cap \mathscr{Z}=\{0\}$. here, $\mathscr{N} / \mathscr{M}$ will be of dimension two.

(4.7) ExAmple. Let $\mathcal{N}$ be the $\mathbb{R}$-span of $W_{1}, W_{2}, X_{1}, X_{2}, Y_{1}$, $Y_{2}$, and $Z$. Let all non-zero brackets be generated by $\left[W_{i}, X_{i}\right]=Y_{i}$, and $\left[W_{i}, Y_{i}\right]=Z, i=1,2$. Let $\mathscr{L}$ be the $\mathbb{R}$-span of $\left\{W_{1}+\alpha W_{2}+\right.$ $\left.\beta X_{1}+\gamma X_{2}+\xi Z, Y_{1}-\alpha Y_{2}+\eta Z\right\}$ where the real numbers, $1, \alpha, \beta$, $\gamma$ are linearly independent over $\mathbb{Q}$ and satisfy the growth condition (1.2), and $\xi, \eta \in \mathbb{R}$ are arbitrary but fixed. The abelian Lie algebra $\mathscr{L}$ is $(\mathrm{GH})$ on $\Gamma[N, N] \backslash N$ and on $\Gamma Z \backslash N$, but $\mathscr{L} \cap \mathscr{Z}=\{0\}$. Let $\Lambda=Z^{*}$, so the polarizing subalgebra is the ideal $\mathscr{M}$ spanned by $\left\{X_{1}, X_{2}, Y_{1}, Y_{2}, Z\right\}$. However, we can act on $\Lambda$ by $\exp t\left(W_{1}-W_{2}\right)$ to get $\Lambda^{\prime}: Y_{1}-\alpha Y_{2}+\eta Z_{2} \mapsto 0$. And we can act on $\Lambda^{\prime}$ by $\exp s\left(Y_{1}+Y_{2}\right)$ to get $\Lambda^{\prime \prime}: W_{1}+\alpha W_{2}+\beta X_{1}+\gamma X_{2}+\xi Z \mapsto 0$. Thus $\Lambda^{\prime \prime} \in \mathscr{O}_{N}(\Lambda)$, and $\Lambda^{\prime \prime}(\mathscr{L})=\{0\}$. By Lemma on page 368 of [C-R2] $\mathscr{L}$ is not $(\mathrm{GH})$ on $\Gamma \backslash N$.

\section{REFERENCES}

[A-B] L. Auslander and J. Brezin, Uniform distribution in solvmanifolds, Adv. in Math., 7 (1971), 111-144.

[A-G-H] L. Auslander, L. Green, and F. Hahn, Flows on Homogeneous Spaces, Annals of Math. Studies No. 53, Princeton Univ. Press, 1963.

[Brl] J. Brezin, Function theory on metabelian solvmanifolds, J. Funct. Anal., 10 (1972), 33-51.

[Br2] _ Harmonic Analysis on Compact Solvmanifolds, Lecture Notes in Math., Vol. 602, Springer-Verlag, Berlin-Heidelberg-New York, 1977.

[Ce-Ro] A. Cerezo and F. Rouvière, Solution élèmentaire d'un opérateur différentiel linéaire invariant a gauche sur un groupe de Lie rél compact et sur un espace homogène réductif compact, Ann. Sci. École Norm. Sup. (4), 2 (1969), 562581.

[C-R1] J. Cygan and L. Richardson, Global solvability on two-step compact nilmanifolds, Trans. Amer. Math. Soc., 279 (1983), 537-554.

[C-R2] _ Globally hypoelliptic systems of vector fields on nilmanifolds, J. Funct. Anal., 77 (1988), 364-371.

[C-R3] _ Global solvability on compact nilmanifolds of three or more steps, Trans. Amer. Math. Soc., 301 (1987), 343-373. 
[F-S] G. B. Folland and E. M. Stein, Hardy Spaces on Homogeneous Groups, Mathematical Notes 28, Princeton Univ. Press, 1982.

[G] R. Goodman, One-parameter groups generated by operators in an enveloping algebra, J. Funct. Anal., 6 (1970), 218-236.

[G-W1] S. Greenfield and N. Wallach, Global hypoellipticity and Liouville numbers, Proc. Amer. Math. Soc., 31 (1972), 112-114.

[G-W2] _ Globally hypoelliptic vector fields, Topology, 12 (1973), 247-253.

[G-W3] _ Remarks on global hypoellipticity, Trans. Amer. Math. Soc., 183 (1973), 153-164.

[H-N] B. Helffer and J. Nourrigat, Caractérisation des opérateurs hypoelliptiques homogènes invariants à gauche sur un groupe de Lie nilpotent gradué, Comm. Partial Differential Equations, 4 (1979), 899-958.

[H] R. Howe, On Frobenius reciprocity for unipotent algebraic groups over $\mathbb{Q}$, Amer. J. Math., 93 (1971), 163-172.

[K] A. A. Kirillov, Unitary representations of nilpotent Lie groups, Uspehi Mat. Nauk, 17 (1962), 57-110; English transl., Russian Math. Surveys, 17 (1962), 53-104.

[M] C. C. Moore, Decomposition of unitary representations defined by discrete subgroups of nilpotent groups, Annals of Math., 82 (1965), 146-182.

[M-W] C. C. Moore and J. Wolf, Square-integrable representations of nilpotent groups, Trans. Amer. Math. Soc., 185 (1973), 445-462.

[R1] L. Richardson, Decomposition of the $L^{2}$-space of a general compact nilmanifold, Amer. J. Math., 93 (1971), 173-190.

[R2] _ Poisson summation on Kirillov orbits, Math. Ann., 239 (1979), 229240.

[R3] _ $N$-step nilpotent Lie groups with flat Kirillov orbits, Colloquium Mathematicum, 52 (1987), 285-287.

[R-S] L. P. Rothschild and E. M. Stein, Hypoelliptic differential operators on nilpotent groups, Acta Math., 137 (1976), 247-320.

[S] L. Schwartz, Théorie des Distributions, Hermann, Paris, 1966.

[S-W] E. M. Stein and G. Weiss, Introduction to Fourier Analysis on Euclidean Spaces, Princeton Univ. Press, Princeton, N. J., 1971.

[W] G. Warner, Harmonic Analysis on Semi-simple Lie Groups I, SpringerVerlag, New York, 1972.

Received February 14, 1989 and in revised form October 13, 1989. Research by the first author was supported in part by Louisiana Education Quality Support Fund contract number 86-LBR-016-04 and by the National Science Foundation. The second author's research was supported in part by the National Science Foundation.

Louisiana State University

BATON ROUGE, LA 70803 


\section{PACIFIC JOURNAL OF MATHEMATICS EDITORS}

V. S. VARADARAJAN

(Managing Editor)

University of California

Los Angeles, CA 90024-1555-05

Herbert Clemens

University of Utah

Salt Lake City, UT 84112

THOMAS ENRIGHT

University of California, San Diego

La Jolla, CA 92093

\section{R. FINN}

Stanford University

Stanford, CA 94305

HermanN FlaschKa

University of Arizona

Tucson, AZ 85721

VAUGHAN F. R. JoNES

University of California

Berkeley, CA 94720

STEVEn KeRCKHOFF

Stanford University

Stanford, CA 94305
C. C. MOORE

University of California

Berkeley, CA 94720

MARTIN SCHARLEMANN

University of California

Santa Barbara, CA 93106

Harold STARK

University of California, San Diego La Jolla, CA 92093

\section{ASSOCIATE EDITORS}

R. ARENS

E. F. BECKENBACH (1906-1982)
B. H. NeumanN

\section{SUPI}

UNIVERSITY OF ARIZONA

UNIVERSITY OF BRITISH COLUMBIA

CALIFORNIA INSTITUTE OF TECHNOLOGY

UNIVERSITY OF CALIFORNIA

MONTANA STATE UNIVERSITY

UNIVERSITY OF NEVADA, RENO

NEW MEXICO STATE UNIVERSITY

OREGON STATE UNIVERSITY
F. WolF
K. YoshidA
(1904-1989)

UNIVERSITY OF OREGON UNIVERSITY OF SOUTHERN CALIFORNIA

STANFORD UNIVERSITY

UNIVERSITY OF HAWAII

UNIVERSITY OF TOKYO

UNIVERSITY OF UTAH

WASHINGTON STATE UNIVERSITY

UNIVERSITY OF WASHINGTON 


\section{Pacific Journal of Mathematics}

Vol. 147, No. $1 \quad$ January, 1991

Mark S. Ashbaugh, Evans Malott Harrell, II and Roman Svirsky, On minimal and maximal eigenvalue gaps and their causes $\ldots \ldots \ldots \ldots \ldots 1$

Robert Coleman and Francis Oisin McGuinness, Rational formal group

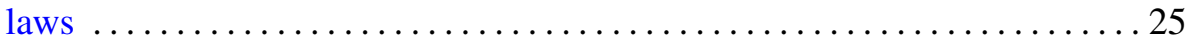

Jacek M. Cygan and Leonard Frederick Richardson, $D$-harmonic distributions and global hypoellipticity on nilmanifolds ...........29

Satya Deo and Kalathoor Varadarajan, Some examples of nontaut

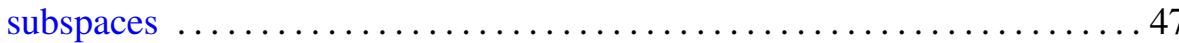

Maria Fragoulopoulou, Automatic continuity of *-morphisms between

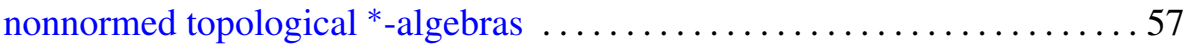

Stephen J. Gardiner, Removable singularities for subharmonic functions . . . 71 Herbert Paul Halpern, Victor Kaftal and László Zsidó, Finite weight projections in von Neumann algebras $\ldots \ldots \ldots \ldots \ldots \ldots \ldots \ldots \ldots \ldots$

Telemachos E. Hatziafratis, Explicit $\bar{\partial}$-primitives of Henkin-Leiterer kernels on Stein manifolds

Ka Hin Leung, A construction of an ordered division ring with a rank one

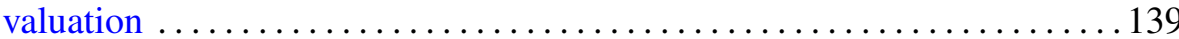

Christopher K. McCord, Nielsen numbers and Lefschetz numbers on solvmanifolds

Katsuro Sakai and Raymond Y. T. Wong, Manifold subgroups of the homeomorphism group of a compact $Q$-manifold

Caroline Perkins Sweezy, $L$-harmonic functions and the exponential square class 